\title{
Dolutegravir plus lamivudine as simplification dual therapy in virologically suppressed HIV-1 infected subjects \\ Laura Comi ${ }^{1 *}$, Elisa Di Filippo', Franco Maggiolo \\ 'Division of Infectious Diseases, ASST Papa Giovanni XXIII, Bergamo, Italy
}

\section{Article Info}

\section{Article Notes}

Received: January 20, 2018

Accepted: February 24, 2018

\section{${ }^{*}$ Correspondence:}

Dr. Laura Comi, Division of Infectious Diseases, ASST Papa Giovanni XXIII, Bergamo, Italy;

E-mail: lalla.comi@gmail.com

c 2018 Comi L. This article is distributed under the terms of the Creative Commons Attribution 4.0 International License.

\section{Keywords}

Dolutegravir

Lamivudine

Dual therapy

INSTI

Virological failure

Maintenance therapy

\section{ABSTRACT}

Introduction: The use of combination antiretroviral therapy (cART) containing three active drugs from at least two different classes is the standard of care for HIV treatment worldwide. The availability of newer drugs with improved potency and tolerability and higher barrier to the development of resistance allows exploring the feasibility of ARV-sparing strategies, namely dual therapies. A dual therapy based on dolutegravir plus lamivudine could be an intriguing simplification strategy for individuals with stable HIV suppression on CART.

Results: Seven studies of dual therapy regimens based on dolutegravir plus lamivudine were critiqued. All of them report a low rate of therapeutic failure due to any cause and a small number of virologic failures. More important virologic failures were not associated with loss of future option as no resistance inducing mutation to ongoing drugs emerged. On the safety side, after the switch, very few short-term adverse events leading to treatment discontinuation were observed and surrogate markers of long term toxicities such as changes in lipid profile and renal function were minimally influenced or improved.

Discussion: Dolutegravir plus lamivudine as a switch option in patients with sustained viral control is still to be considered an experimental approach. Although small in number and heterogeneous in nature the studies that evaluated the effectiveness of dolutegravir plus lamivudine dual therapy have documented substantial virologic efficacy and tolerability of the regimen without exposing patients to the risk of selecting for INSTI-inducing resistance mutations.

\section{Introduction}

The use of combination antiretroviral therapy (cART) containing three active drugs from at least two different classes began in the mid-1990s, and since then has been the standard of care for HIV treatment worldwide. Current HIV treatment guidelines recommend first line anti-retroviral (ARV) regimens consisting of two nucleoside reverse transcriptase inhibitors as a backbone combined with a third agent from either the non-nucleoside reverse transcriptase inhibitor, or the boosted protease inhibitor (PI), or the integrase strand transfer inhibitor (INSTI) classes ${ }^{1-2}$. Advances in the virologic and safety profiles of antiretroviral drugs have created opportunities to investigate alternative approaches. The availability of newer drugs with improved potency and tolerability and higher barrier to the development of resistance allows exploring the feasibility of 
ARV-sparing strategies, namely dual therapies. Potential advantages of two-drug regimens include reduced longterm toxicity, complexity, and costs ${ }^{3}$. This approach may result particularly suited for maintenance therapy in patients with steadily controlled viral replication, who wish or need to simplify cART.

In these settings, several experiences of 2-drugs regimens based on a boosted PI plus lamivudine (3TC) have demonstrated favorable results in terms of efficacy, but are somehow penalized by metabolic side effects and risk for drug-drug interactions ${ }^{4-5}$.

Dolutegravir (DTG) is a potent INSTI, exhibiting a rapid reduction in viral load (VL) and a high barrier to resistance. DTG is a once daily drug, well tolerated, that can be taken with or without food, with a low potential for drug-drug interactions and a high genetic barrier. Maintenance therapy with DTG plus rilpivirine was comparable to standard triple cART in largely randomized trials ${ }^{6}$. A possible alternative and intriguing simplification strategy for individuals with stable HIV suppression on cART is the use of a dual therapy based on dolutegravir plus lamivudine, a potent cytidine nucleoside analogue without major side effects, and that has a well-proven safety profile ${ }^{7}$.

In this paper, we review all available data of the DTG+3TC combination in pre-treated HIV positive patients in order to offer a comprehensive evaluation of this option that is already used in clinical practice although it should still be considered as experimental.

\section{Methods}

We searched Medline, Embase, Cochrane central and web of science, as well as abstract of major HIV conferences for studies which included HIV-infected individuals with undetectable viral load on triple antiretroviral therapy who switched to DGT+3TC dual therapy.

\section{Studies in naive patients}

DTG+3TC is an investigational 2-drug regimen potential for co-formulation into a once daily single tablet regimen with lower cART $\operatorname{cost}^{8}$. In the proof-of-concept PADDLE study ${ }^{9}, 18 / 20$ naïve patients, with baseline HIV-RNA $<100,000$ copies/ml and CD4 count > 200 cells $/ \mathrm{mcL}$, had a viral load < 50 copies/ml 96 weeks after starting DTG+3TC dual therapy. In the ACTG 5353 pilot study 120 naïve subjects received DTG+3TC and were virologically evaluated by FDA snapshot analysis after 24 weeks $^{10}$. Virologic efficacy was demonstrated in $108 / 120$ patients. Three patients experienced virologic failure and one of them selected for the $\mathrm{M} 184 \mathrm{~V}$ reverse transcriptase and the R263K integrase mutations.

\section{Studies in experienced patients}

\section{Efficacy analysis in maintenance therapy}

Overall DTG+3TC in switch studies demonstrated a low rate of therapeutic failure for any cause and a very low rate of virologic failures (table 1).

So far only two randomized studies on DTG+3TC simplification have been published. Blanco and Colleagues, enrolled in an open label, non inferiority, randomized controlled trial (DOLAM study) ${ }^{11} 91$ HIV infected adults on stable triple ART. All of them had a viral load of less than 50 copies/ml in the last year, no prior viral failure or resistance mutations to 3TC/FTC or INSTI, and a negative HBsAg. They were randomized 1:1:1 to continue triple cART (control), or switch to either DTG+3TC or DTG monotherapy. After 24 weeks of follow-up, 3 patients prematurely discontinued

Table 1: Summary of studies on DTG+3TC in experienced patients.

\begin{tabular}{|c|c|c|c|c|c|c|c|}
\hline Study & Type & Country & $\begin{array}{l}\text { Patients on } \\
\text { DTG+3TC }\end{array}$ & $\begin{array}{c}\text { Number (median)/ } \\
\text { Time (years) on } \\
\text { previous ARV }\end{array}$ & $\begin{array}{c}\text { Presence of } \\
\text { mutations prior to } \\
\text { switch }\end{array}$ & $\begin{array}{l}\text { Virological } \\
\text { failure }\end{array}$ & $\begin{array}{c}\text { Discontinuation } \\
\text { due to adverse } \\
\text { events/ any }\end{array}$ \\
\hline DOLAM [11] & $\begin{array}{l}\text { Open-label random- } \\
\text { ized trial }\end{array}$ & Spain & 29 & $\begin{array}{c}/ \\
>1 \text { years }\end{array}$ & NO & $\begin{array}{c}1 \\
\text { No resistance }\end{array}$ & 0 \\
\hline $\begin{array}{l}\text { Taiwo } \\
{[12]}\end{array}$ & $\begin{array}{l}\text { Randomized clinical } \\
\text { trial }\end{array}$ & USA & 44 & $\begin{array}{c}/ \\
5.7 \text { years }\end{array}$ & NO & 1 & $1 / 2$ \\
\hline LAMIDOL [13] & Single arm trial & France & 104 & $\begin{array}{c}/ \\
4 \text { years }\end{array}$ & NO & $\begin{array}{c}1 \\
\text { No resistence }\end{array}$ & $1 / 3$ \\
\hline DOLULAM [14] & Prospective cohort & France & 27 & $\begin{array}{c}/ \\
17 \text { years }\end{array}$ & YES & 0 (2 blips) & $0 / 3$ \\
\hline Maggiolo [16] & Prospective, cohort & Italy & 203 & $\begin{array}{c}3 / \\
10.3 \text { years }\end{array}$ & NO & 0 & $5 / 12$ \\
\hline Borghetti [17] & $\begin{array}{l}\text { Retrospective, } \\
\text { cohort }\end{array}$ & Italy & 183 & $\begin{array}{c}\text { / } \\
9 \text { years }\end{array}$ & YES & $\begin{array}{c}3 \\
\text { No resistence }\end{array}$ & $11 / 21$ \\
\hline $\begin{array}{l}\text { Hidalgo- Tenorio } \\
\text { [18] }\end{array}$ & $\begin{array}{l}\text { Retrospective } \\
\text { cohort }\end{array}$ & Spain & 105 & $\begin{array}{c}3 / \\
13 \text { years }\end{array}$ & Not reported & 0 (2 blips) & 0 \\
\hline Yagci Caglayik [19] & $\begin{array}{l}\text { Retrospective } \\
\text { cohort }\end{array}$ & Turkey & 32 & $\begin{array}{c}3 / \\
13 \text { years }\end{array}$ & Not reported & 0 & 0 \\
\hline
\end{tabular}


due to low-level viral failure: 1 patient in the dual group, without the emergence of resistance conferring mutations, and 2 patients in the dolutegravir monotherapy arm, with evidence of resistance mutations. As a consequence, the Data Safety Monitoring Board recommended stopping DTG monotherapy and allowed the DTG+3TC arm to be continued.

The second study, recently published by Taiwo. et $\mathrm{al}^{12}$, is a pilot randomized clinical trial in which 90 HIV infected adults, virologically suppressed on stable triple ARV, without history of virologic failure and negative hepatitis B surface antigen were randomized to continue triple ART (45 pts) or switch to DTG+3TC (44 pts).After 48 weeks of follow-up, only 1 patient in the DTG $+3 \mathrm{TC}$ arm showed an HIVRNA > $50 \mathrm{cp} / \mathrm{ml}$, without the emergence of viral mutations.

The LAMIDOL trial ${ }^{13}$ is an open label, single arm trial, in which 110 virologically suppressed patients, on a first line cART based on a classic triple drug combination were enrolled in 19 Clinics in France. They were first switched to dolutegravir as a substitute for the third agent and then, after 8 weeks, received DTG+3TC. One hundred and four patients started DTG+3TC: of those $86 \%$ were males, $70 \%$ MSM with a median age of 45 years and a median time since HIV diagnosis of 6.3 years (2.3-24.5). At week 48 of follow-up 3 therapeutic failures, only one due to virological failure, were observed.

Most patients enrolled in all the above-mentioned studies were on their first or second-line therapies. However, most of the data on the DTG+3TC strategy have been generated by clinical cohorts, which comprehended heavily pre-treated patients on ART for a longer time, in some cases longer than 10-15 years and with a median of 3 different antiretroviral regimens prior to the switch.

A small prospective cohort (DOLULAM) ${ }^{14}$ enrolled 27 patients that had been taking cART for a median of 215 months (IQR 22-239) and the last therapy for a median of 51 (IQR 13-108) months. All patients received dolutegravir plus lamivudine, even if ten of them had evidence of M184V mutation in a previous genotypic test prior switch with the evidence of M184V mutation. No virological failure was reported after a median follow-up time of 96 weeks.

An Italian prospective, multi-center, cohort study, enrolled 203 patients that were on stable cART, with a confirmed viremia $<50$ copies $/ \mathrm{ml}$ in the last six months, in the absence of M184V mutation or HBsAg positivity ${ }^{15-16}$. All needed to switch therapy due to clinically relevant reasons such as concomitant diseases, altered laboratory tests, drug adverse events or risk of drug-to-drug interactions.

Patients enrolled were mostly men (75.4\%) with a median age of 52 years (IQR 47-58). At switch patients were on ARV drugs for a median of 10.3 years (IQR 5.5-17-
6), virologically suppressed for a median of 72 months (IQR 33-121) and experienced a median of three therapeutic lines.

All subjects were prospectively followed up to week 48 , and all remained on the dual therapy during the whole period. Neither virological failure nor viral blip was detected ${ }^{15}$. According to a more recent report with extended follow-up, 12 patients stopped therapy after week $48^{16}$.

Another Italian cohort study retrospectively evaluated 494 cART-experienced and virologically suppressed HIV positive patients, according to different simplification strategies (lamivudine+boosted PIs versus DTG+3TC). One-hundred eighty-three subjects received dolutegravir, 170 switched to darunavir and 141 to atazanavir. Groups differed for age, HIV risk-factor, time since HIV diagnosis, time on antiretroviral therapy, previous cART regimen and reasons for switching ${ }^{17}$.

Time to treatment discontinuation, due to any reason, and virological failure were the endpoints compared by Kaplan-Meier estimator. Probabilities of remaining on the considered treatment at week 48 and 96 were respectively: $79.8 \%$ and $48.3 \%$ for darunavir, $87 \%$ and $70.9 \%$ for atazanavir, $88.2 \%$ and $82.6 \%$ for dolutegravir. Moreover, among patients who discontinued, virological failure was the cause in 3/21 cases in the dolutegravir group, 6/123 in the darunavir group and 4/97 in the atazanavir group ${ }^{17}$.

Furthermore, a recent Spanish cohort ${ }^{18}$ reported 105 patients treated for a median of 13 years with a median of 3 different cART combinations. Baseline HIV-RNA was $<50$ copies $/ \mathrm{ml}$ in $96.2 \%$ of cases, and they had a median CD4 count of 732 cells/mcL. The most frequent reasons for the switch were simplification (39\%) and toxicity (44.8\%). Eighty-four of them performed at least an HIV-RNA test after the therapeutic switch to DTG+3TC and over a median follow-up of 23 weeks. Viral load was $<50$ copies $/ \mathrm{ml}$ in 97.2 subjects, showing the last 2 individuals viral blips of 55 and 239 copies/ml, respectively.

Finally, in a small Turkish cohort study ${ }^{19}, 32$ patients were included from 6 centers. Twenty-nine patients were virologically suppressed before switch, three patients had HIV-RNA levels 21503, 656 and 59 copies/mL. No patient experienced virologic failure with prior regimens. CD4 T cell counts were $>200$ cells $/ \mathrm{mm}^{3}$ in all patients, except one $\left(90\right.$ cells $/ \mathrm{mm}^{3}$ ) before switch. No patient experienced serious adverse events, AIDS-related events, or died during the follow-up period. Authors defined DTG+3TC combination as efficient and well tolerated, and did not report any virologic failure.

\section{Safety analysis in maintenance therapy}

The main rationale for dual therapy is to optimize health status and quality of life without compromising 
control of HIV infection. Very few short-term adverse events leading to treatment discontinuation were observed in DTG+3TC studies. No patient stopped therapy because of an adverse event in the Spanish cohort ${ }^{18}$, in the DOLAM study $^{11}$, or in a Turkish cohort ${ }^{19}$ in which 32 patients were switched to DTG-3TC because of documented renal toxicity or renal impairment, hyperlipidemia, adverse events with current cART, prevention of potential toxicities and regimen simplification. In a recent update of a previously described cohort ${ }^{16}, 5 / 203$ patients discontinued treatment for intolerance, being muscle aches the most common cause. In the same cohort 3 patients died, but because of underlying diseases unrelated to HIV or therapy ${ }^{16}$. In the LAMIDOL trial, ${ }^{13} 8 / 110$ patients stopped therapy because of an adverse event and 4 of them were possibly related to DTG use. As a whole, studies on DTG+3TC did not report a relevant occurrence of CNS related adverse events.

There are a few data about long-term adverse effects of DTG + 3TC and most of them are based on surrogate markers such as changes in lipid profile and renal function. Both the Italian cohorts investigated the metabolic assets of patients after the switch with substantially similar results ${ }^{15,17}$. The authors registered a decrease in median triglycerides and total cholesterol, an increment of HDLcholesterol and a reduction of median LDL-cholesterol. Changes were, however, limited: total cholesterol $-7 \mathrm{mg} /$ $\mathrm{dL}(\mathrm{p}=0.047)$; LDL-cholesterol $-7 \mathrm{mg} / \mathrm{dL}(\mathrm{p}=0.335)$, HDLcholesterol $+4 \mathrm{mg} / \mathrm{dL}(\mathrm{p}=0.047)$ and triglycerides $-31 \mathrm{mg} /$ $\mathrm{dL}(\mathrm{p}=0.012)$ in the first cohort [16] and total cholesterol $-24 \mathrm{mg} / \mathrm{dL}(\mathrm{p}<0.01) ;$ LDL-cholesterol - $15 \mathrm{mg} / \mathrm{dL}$ $(\mathrm{p}=0.009)$ and triglycerides $-66 \mathrm{mg} / \mathrm{dL}(\mathrm{p}<0.001)$ in the second cohort ${ }^{14}$. The clinical implications of these results are still to be elucidated.

Kidney function was evaluated in both Italian cohort ${ }^{15-17}$ and in the DOLULAM study ${ }^{14}$ and the dual combination was not associated with a decline in renal function. However, it must be noted that a relevant proportion of subjects included in these studies were previously exposed to tenofovir whose withdrawal could partially influence the outcome.

\section{Discussion}

Dual antiretroviral therapy seeks to reduce toxicities and improve quality of life by reducing the drug burden. Toxicities associated with antiretroviral agents are often drug class specific. Nucleoside analogs use has been commonly associated with mitochondrial toxicity (lipoathrophy, functional kidney dysfunction, cardiovascular accidents, and osteoporosis) $)^{20,21}$.

So far, however, the need of maintaining the efficacy and convenience of robust cART limited clinicians' options.

Dolutegravir, similarly to boosted-darunavir, is characterized by a high affinity to its target, resulting in strong and sustained binding ${ }^{22}$. As a consequence, in vitro selection of mutants resistant to dolutegravir is very difficult. To date, no emergent dolutegravir-resistant virus has ever been reported in a patient in whom dolutegravir was prescribed as a first $\mathrm{INI}^{23}$.

Dolutegravir plus lamivudine as a switch option in patients with sustained viral control is still to be considered an experimental approach, that finds a contraindication in chronic hepatitis B co-infected patients. So far, 7 DTG+3TC switch studies evaluated the effectiveness of this dual therapy regimen.

Although small in numbers and heterogeneous in nature these experiences have documented a substantial virologic efficacy and tolerability of the dual regimen without exposing patients to the risk of selecting for INSTIinducing resistance mutations.

Two randomized study (DOLAM study and ASPIRE study) ${ }^{11,12}$ provide a preliminary comparative evidence that virologic suppression is similar to that obtained with standard cART. In these studies, only 2 patient (one in each study) discontinued due to low-level viral failure and without losing future options as, at failure, there was no evidence of induced resistance mutations. The other studies support this result.

The dual therapy is substantially well tolerated without specific signature adverse events and with evidence of a neutral effect on lipid parameters and on kidney function.

Altogether, these data suggest that a dual therapy with DTG+3TC in treatment experienced and virologically suppressed patients contributes to set up the new paradigm of induction-maintenance in the management of HIV treatment ${ }^{24}$. Up to date, however, the scattered nature of data, the type of studies, mostly observational, and the limited experience both in terms of number of treated subjects and on time on active follow-up do not allow to make a definitive point about how much the dual therapeutic option would be advantageous for chronically infective patients. This is a limit of the present review, too.

Finally, dual therapy with dolutegravir plus lamivudine could also be an option to contain therapeutic costs, which is an aspect of increasing relevance worldwide. Using a mathematical simulation, a group of American researchers projected the clinical and economic outcomes of 4 different therapeutic strategies over a period of five years. They demonstrated that DTG + 3TC could result in cost-effective allowing for a global reduction of more than 500 million dollars in cART costs in the United States over the considered time period ${ }^{8}$.

Despite the fact that the newest dual regimens such as DTG+3TC could save money, reduce toxicity and spare drug options for the future, the observational and nonrandomized nature of most studies, the limited number of 
subjects treated and the limited follow-up should induce caution when this approach is used in clinical practice. A large, worldwide, randomized, clinical study involving 550 experienced patients has recently started recruitment and will hopefully reframe the picture in the near future ${ }^{25}$.

\section{References}

1. European AIDS Clinical Society (2017) Guidelines: Clinical management of treatment of HIV infected adults in Europe. Version 9.0. October 2017

2. Huldrych F, Günthard MD, Michael S. Saag MD, et al. Antiretroviral Drugs for Treatment and Prevention of HIV Infection in Adults 2016 Recommendations of the International Antiviral Society-USA Panel. JAMA. 2016; 316(2): 191-210.

3. Baril JG, Angel JB, Gill MJ, et al. Dual therapy treatment strategies for the management of patients infected with HIV: a systematic Review of current evidence in ARV-Naive or ARV-experienced, virologically suppressed patients. PLoS One. 2016 Feb 5; 11(2): e0148231. doi: 10.1371/journal.pone.0148231.

4. Di Giambenedetto S, Fabbiani M, Colafigli M, et al. Safety and feasibility of treatment simplification to atazanavir/ritonavir + lamivudine in HIV-infected patients on stable treatment with two nucleos(t) ide reverse transcriptase inhibitors + atazanavir/ritonavir with virological suppression (Atazanavir and Lamivudine for treatment Simplification, AtLaS pilot study). J Antimicrob Chemother. 2013 Jun; 68(6): 1364-72. doi: 10.1093/jac/dkt007. Epub 2013 Jan 30.

5. De Luca A, Doino M, Fabbiani M, et al. Treatment simplification to atazanavir/ritonavir plus lamivudine qd in patients on two NRTIs plus atazanavir/ritonavir with optimal virologic control: 48 weeks safety and efficacy results from a pilot study (atazanavir and lamivudine sim-plification study). 6th International AIDS Society Conference on HIV Pathogenesis, Treatment and Pre-vention. 2011 July 17 - 20; Rome, Italy.

6. Libre JM, Hung CC, Brinson C, et al. Phase III SWORD 1\&2: switch to DTG + RPV maintains virologic suppression through 48 weeks (abstract 44LB). In: Program and abstracts of the 2017 Conference on Retroviruses and Opportunistic Infections. Seattle. February 13-16, 2017.

7. Arribas JR, Girard PM, Landman R. Dual treatment with lopinavir ritonavir plus lamivudine versus triple treatment with lopinavir ritonavir plus lamivudine or emtricitabine and a second nucleos $(\mathrm{t})$ ide reverse transcriptase inhibitor for maintenance of HIV-1 viral suppression (OLE): a randomised, open-label, non-inferiority trial. Lancet Infect Dis. 2015. Available at: http://dx.doi.org/10.1016/ S1473-3099(15)70129-5. Accessed 1 Oct 2016

8. Girouard MP, Sax PE, Parker RA, et al. The Cost-effectiveness and Budget Impact of 2-Drugs Dolutegravir-Lamivudine Regimens for the Treatment of HIV Infection in the United States. Clin Infect Dis, 2016; 62: 748-791.

9. Figueroa MI, Rolon MJ, Patterson P, et al. Dolutegravir-lamivudine as initial therapy in HIV-infected, ARV naive patients (poster abstract MOPEB028): 96 week results of the PADDLE trial. International AIDS Conference 2017, Paris. July 23-26, 2017.

10. Taiwo BO. ACTG A5353: A pilot study of dolutegravir plus lamivudina for initial treatment oh HIV-1-infected participants with HIV-RNA < 500.000 copies/mL. Clin Infect Dis, cix1083, doi.org/10.1093/cid/ cix1083 (Epub ahead of print)
11. Blanco JL, Rojas J, Negredo E, et al. for the DOLAM Study Group. Planned 24-week Analysis of two Dolutegravir (DTG).based Simplification Strategies (abtract PS1/3). 16th European AIDS conference, Milan. October 25-27, 2017

12. Taiwo BO, Marconi VC, Berzins B, et al. Dolutegravir plus lamivudine maintain HIV-1 suppression through week 48 in a pilot randomized trial. Clin Infect Dis. 2017 Dec 26; doi: 10.1093/cid/cix1131. (Epub ahead of print)

13. Joly V, Burdet C, Raffi F, et al. Promising Results of Dolutegravir + lamivudina Maintenanace in ANRS167 LAMIDOL Trial (abstract PE 9/11). 16th European AIDS conference, Milan. 2017 October; 25-27.

14. Reynes J, Meftah N, Tuaillon E, et al. Dual regimen with dolutegravir and lamivudine maintains virologic suppression even in heavily treatment experienced HIV-infected patients: 96 weeks results from mantainance DOLULAM study (abstract 2755). 9th IAS Conference on HIV Science (IAS 2017) Paris. 2017 July; 23-26.

15. Maggiolo F, Gulminetti R, Pagnucco L, et al. Lamivudine/dolutegravir dual therapy in HIV-infected, virologically suppressed patients. BMC Infect Dis. 2017; 17: 215. doi: 10.1186/s12879-017-2311-2.

16. Maggiolo F, Gulminetti R, Pagnucco L, et al. Durability of dolutegravir + lamivudine as simplification cART in patients with suppressed HIVRNA (abstract PE 9/49). 16th European AIDS conference, Milan. 2017 October; 25-27.

17. Borghetti A, Moschese D, Baldin G, et al. Efficacy and tolerability of lamivudine plus dolutegravir compared with lamivudine plus boosted PIs in HIV positive, virologically suppressed individuals from the clinical practice (abstract PE 9/76). 16th European AIDS conference, Milan. 2017 October; 25-27.

18. Hidalgo-Tenorio C, de Jesus SE, Santos J, et al. Multicenter study of the effectiveness and safety of a dual therapy with dolutegravir plus lamivudine in treatment-experienced HIV-patients. (abstract PE 9/68) $16^{\text {th }}$ European AIDS conference, Milan. 2017 October; 25-27.

19. Yagci Caglayik D, Gokengin D, Inan A, et al. Real life experience of dolutegravir and lamivudine dual therapy as a switching regimen in HIV-TR cohort. (abstract PE 9/12) $16^{\text {th }}$ European AIDS conference, Milan. 2017 October; 25-27.

20. Cote HC, Brumme ZL, Craib KJ, et al. Changes in mitochondrial DNA as a marker of nucleoside toxicity in HIV-infected patients. N Engl J Med. 2002; 346: 811-820.

21. Jones R, Sawleshwarkar S, Michailidis C, et al. Impact of antiretroviral choice on hypercholesterolaemia events: the role of the nucleoside reverse transcriptase backbone. HIV Med. 2005; 6: 396-402.

22. Walmsley S, Antela A, Clumeck N, et al. on behalf of the SINGLE investigators. Dolutegravir plus abacavir/lamivudine for the initial treatment of HIV-1 infection. N Engl J Med. 2013; 369: 1807-1818.

23. Wainberg MA, Han YS. Will drug resistance against dolutegravir in initial therapy ever occur. Front Pharmacol 2015; 6: 90.

24. Soriano V. Dual antiretroviral therapy for HIV infection. Expert Opin Drug Saf. 2017; 16(8): 923-932.

25. Pottage JC. A phase III, randomized, multicenter, parallel-group, noninferiority study evaluating the efficacy, safety and tolerability of switching to dolutegravir plus lamivudine in HIV 1 infected adults who are virologically suppressed https://www.viivhealthcare.com/ media/press-releases/2018. Last accessed 20/feb/2018. 